\title{
Early and contemporary human neuroimaging studies of serotonergic psychedelics
}

\author{
Enzo Tagliazucchi \\ University of Buenos Aires and Buenos Aires Physics Institute, National Scientific and Technical \\ Research Council (CONICET), Pabellón I, Ciudad Universitaria (1428), Buenos Aires, Argentina
}

\begin{abstract}
Serotonergic psychedelics are known to elicit changes in conscious awareness, including perception of the environment and the self, as well as in mood, emotion and different aspects of cognition (Nichols, 2016). The effect of these compounds is complex and resists a straightforward classification that is useful for other drugs, such as "stimulants" or "sedatives". While the effects of certain psychedelics do have a stimulant dimension, their defining characteristic is the capacity to temporarily induce a state of altered consciousness. Because of this, the study of psychedelics cannot be based only on animal models, since humans are alone in their capacity to explicitly report the contents of their conscious awareness. Psychedelic research with healthy human subjects necessitates techniques for the non-invasive recording of brain activity or its physiological and metabolic correlates. These techniques are referred to as "neuroimaging", and here we review their application in the study of the neural correlates of altered consciousness induced by serotonergic psychedelics.
\end{abstract}

Keywords: Psychedelics; neuroimaging; consciousness

\section{Introduction and scope}

Serotonergic psychedelics are known to elicit changes in conscious awareness, including perception of the environment and the self, as well as in mood, emotion and different aspects of cognition (Nichols, 2016). The effect of these compounds is complex and resists a straightforward classification that is useful for other drugs, such as "stimulants" or "sedatives". While the effects of certain psychedelics do have a stimulant dimension, their defining characteristic is the capacity to temporarily induce a state of altered consciousness. Because of this, the study of psychedelics cannot be based only on animal models, since humans are alone in their capacity to explicitly report the contents of their conscious awareness. Psychedelic research with healthy human subjects necessitates techniques for the non-invasive recording of brain activity or its physiological and metabolic correlates. These techniques are referred to as "neuroimaging", and here we review their application in the study of the neural correlates of altered consciousness induced by serotonergic psychedelics.

Psychedelics share agonism at the serotonin $5-\mathrm{HT}_{2 \mathrm{~A}}$ receptor as a common mechanism of action, and are found in two different families of chemical compounds. Substituted tryptamines include psilocybin, psilocin, and dimethyltryptamine (DMT), and substituted phenethylamines include mescaline and its 
analogues. The chemical structure of lysergic acid diethylamide (LSD) includes both the tryptamine and phenethylamine moieties and belongs to the ergotamines (Nichols, 2016). These particular compounds (psilocybin, DMT, mescaline and LSD) are frequently called "classic psychedelics", since they were encountered by Western scientific mainstream before the proliferation of hundreds of synthetic psychedelics with similar effects and mechanisms of action (Shulgin et al., 2011). The classic psychedelics are extremely safe drugs and thus are the most frequently investigated in human neuroimaging experiments. The scope of this review is limited to experiments using these compounds, with the understanding that virtually no studies of interest have been left out due to the popularity of classic psychedelics in human neuroimaging experiments.

In terms of experimental techniques, the present review adopts a broad definition of what constitutes neuroimaging, including functional magnetic resonance imaging (fMRI), positron emission tomography (PET) and single-photon emission computed tomography (SPECT), but also electroencephalography (EEG) and magnetoencephalography (MEG). While these last two methods are frequently not considered neuroimaging techniques, advances in source imaging algorithms allow them to perform relatively accurate localization of source activity, justifying their inclusion in this review (Michel, 2004).

This review beings with a brief overview of early studies of serotonergic psychedelics, mainly performed in the decades of 1950s 1960s and using EEG to record brain activity. While advances in neuroimaging methods and data analyses algorithms have rendered these studies for the most part obsolete, some of them anticipated well-established results and thus deserve a place in this review. Afterwards, studies using metabolic imaging (PET and SPECT), fMRI and EEG/MEG are reviewed, including the most common variations in experimental protocols (e.g. resting-state and task-based studies). In recent years, the number of neuroimaging studies using psychedelics has grown considerably. Thus, an exhaustive systematic review of all articles was not attempted; instead, this review aims to review the majority of studies, with focus on those that are considered the most influential or representative. A systematic review of studies (excluding articles using EEG and MEG) up to the year 2016 can be found in dos Santos et al., 2016.

\section{Early EEG studies and their limitations}

Scientific research with psychedelics during the 1950s and 1960s was aligned with the cultural zeitgeist of those decades, revolving mainly around LSD and mescaline. The advantages and idiosyncrasies of psilocybin and DMT (including ayahuasca, the Amazonian DMT-containing concoction) were not recognized and explored until decades after. The vast majority of early studies addressed the EEG correlates of the acute effects of LSD, anticipating an interesting knowledge gap to be discussed later in this review: even though some of most heavily used and abused psychedelics are substituted phenethylamines, virtually no studies addressed the neurophysiological effects of psychedelic phenethylamines, since neuroimaging research has almost systematically excluded mescaline up to the present day.

It is difficult -and most likely unnecessary- to pinpoint the first EEG study of the acute effects of LSD in humans. Early studies converge on two findings that have been replicated by contemporary research: LSD decreases the power of alpha $(8-12 \mathrm{~Hz})$ scalp oscillations, and increases the average peak of the alpha rhythm (Gastaut et al., 1953; Bradley et al., 1953; Bradley and Elkes, 1957; Goldstein et al., 1963). EEG captures electric displacement currents reaching the scalp; these currents are caused by the transport of 
ions across the cell membrane that is required to generate an action potential (neural spike) (Da Silva, 2009). Even though currents generated by a single cell are impossible to measure, those generated by large synchronized cell assemblies can lead to scalp potentials in the range of $1 \mu \mathrm{V}$ (i.e. one millionth of a volt), which can be recorded using EEG. Thus, LSD was shown to affect cell assemblies synchronously activated at a frequency between $8-12 \mathrm{~Hz}$; this is known as the alpha rhythm and it is the landmark feature of eyes-closed resting state human EEG.

Since artefacts of muscular origin are known to increase high-frequency power in EEG recordings, it remains difficult to the present day to determine whether LSD or other psychedelics cause fast oscillations. However, an early study using implanted electrodes in cats was likely to overcome these limitations, finding low-voltage fast cortical EEG, and disorientation, howling, periodic pacing and starring associated with slow-wave high-voltage EEG recordings (Horovitz et al., 1965). Other studies directly addressed the effects of LSD and mescaline in intracranial human EEG recordings, corroborating the decreases in the alpha rhythm, but also finding increased activity in the beta range $(12-30 \mathrm{~Hz})$ in cortical and subcortical regions, predominantly in the caudate nucleus, the amygdala, and the hippocampal and septal areas (Monroe et al., 1957; Monroe and Heath, 1961; Monroe, 1974). These studies display two characteristic features of early research with human subjects: a tendency to consider psychedelics chiefly as psychotomimetic agents (i.e. drugs leading to a transient state of psychosis, as seen in psychiatric diseases such as schizophrenia), and ethical standards that would be inadmissible according to present guidelines.

Early human research with psychedelics and EEG was not limited to the frequency content of resting state EEG, but also addressed the modulation of responses elicited by sensory stimulation. For instance, Rodin and Luby investigated how LSD affected visual evoked potentials arising due to photic stimulation at different frequencies (Rodin and Luby, 1966). They reported the disappearance of the "rhythmic after discharge" and a decrease in amplitude of the major positive component of the evoked potential; however, they did not report changes in the latency of the components. Chapman and Walter found that responses to flashes of light were reduced in amplitude under the acute effects of LSD, with late rhythmic components reduced or abolished (Chapman and Walter, 1965). Inconsistencies were found concerning EEG potentials evoked by ulnar (or cubital) nerve stimulation, with reports of preserved response (Shagass et al., 1962) and increased second negative component (Shagass and Schwartz, 1964). Shagass also reported increased frequency of visually evoked "after rhythms", without correlation between changes in background EEG and those in evoked potentials (Shagass, 1967). This work also reported reduced amplitudes of both somatosensory and visual responses, and earlier onset of the former.

Two studies of high contemporary interest addressed how infusion of LSD altered sleep and, in particular, the EEG patterns indicating the presence of different sleep stages (Muzio et al., 1964; Muzio et al., 1966). These studies can be summarized by the observation that LSD can shift EEG patterns from those indicative of deep sleep / reduced awareness, to those representative of dreaming (rapid eye movement [REM] sleep). These studies are a good example of pioneering human research with psychedelic drugs that should be replicated using contemporary experimental and data analysis techniques. The relevance of this work is manifest in the recent proposal of exploring psychedelics to restore conscious awareness in brain injured patients (Scott and Carhart-Harris, 2019).

The contribution of early EEG studies to our knowledge of the neurophysiological bases of the psychedelic state is difficult to evaluate. On the one hand, many studies anticipated contemporary findings obtained using more advanced experimental methods, such as those concerning the changes in amplitude 
and peak frequency of the alpha rhythm under LSD (Carhart-Harris et al., 2016). Many studies addressed relevant and timely problems that should be revisited and replicated using modern technologies. Other studies produced interesting results that remain to be validated by current research, such as the lateralization of EEG amplitudes under LSD (Goldstein and Stoltzfus, 1973). On the other hand, it is difficult to estimate the number of false positives resulting from inadequate data analysis and experimental design. While we now take computerized data analysis for granted, this was far from the standard during the 1950s and 1960s, as expressed by Rodin and Luby when discussing the status of the field in 1966: "Most studies were carried out by simple visual inspection of the recordings except for that by Grey Walter who used toposcopic analysis and that by Goldstein et al. who used Drohocki's integrator". It should be obvious that simple visual inspection cannot meet the strict methodological and statistical requirements of contemporary scientific research. Perhaps more problematic is experimental design, with several open label and unblinded studies. Expectation effects are strongly suggested, for instance, by a study reporting hypomania and dysphoria induced by $7 \mu$ g of LSD (Greiner et al., 1958), while modern research failed to detect self-reported changes in perception, mentation, or concentration at this dose (Yanakieva et al., 2019). Finally, some of the early studies using LSD in humans should serve as a warning against relaxing ethical standards in current research with serotonergic psychedelics and psychoactive drugs in general.

\section{Metabolism and binding affinity}

Before the development of fMRI in the early 1990s, the most common neuroimaging techniques were based on the direct quantification of metabolism by means of cerebral blood flow and glucose consumption. Imaging methods such as PET and SPECT offer relatively good anatomical localization, but very poor temporal resolution. The static view of brain metabolism provided by these tools is insufficient to investigate dynamic inter-areal synchronization (functional connectivity). However, they provide insights that are often complementary to other imaging techniques, and have been used to establish some long-standing results concerning the neural correlates of the psychedelic state in humans.

Studies using psilocybin, PET and SPECT provided new insights on the neuroanatomical bases of the psychedelic state. Administration of psilocybin led to lobal increases in the cerebral metabolic rate of glucose (CMRglu), especially in regions belonging to the frontomedial and frontolateral cortex, anterior cingulate, and temporomedial cortex (Vollenweider et al., 1997). Some of these changes in CMRglu (assessed with PET) correlated with the dose and the reported subjective effects of the participants. Another study investigating glucose metabolism under psilocybin reported increases regional CMRglu in the right anterior cingulate and right frontal operculum, and decreased regional CMRglu in the thalamus and precentral cortex (Gouzoulis-Mayfrank et al., 1999). The latter correlated with reports of anxiety and depression, while the former correlated positively with stereotyped thoughts.

Cerebral blood flow under the acute effects of oral DMT (ayahuasca) was investigated by Riba et al., and more recently by Sanches et al. The first of these two articles reported that ayahuasca increased activation in the anterior cingulate and the frontal gyrus (right hemisphere), the amygdala and parahippocampal gyrus (left hemisphere), and the anterior insula and inferior frontal gyrus (bilateral) (Riba et al., 2006). The second study, investigating a population of patients suffering from major depression, reported 
increased blood perfusion in the subgenual anterior cingulate which correlated with reductions in depressive and anxiety scores (Sanchez et al., 2016). SPECT was also used to demonstrate increased blood flow in frontal regions under mescaline, which correlated with the intensity of the subjective effects (Hermle et al., 1992).

In many applications, PET has been superseded by fMRI blood-oxygen-level-dependent (BOLD) imaging; however, the use of different radioligands in PET imaging can provide information not available from fMRI experiments alone. The use of a radiolabeled glucose analogue (fludeoxyglucose [FDG]) allows the quantification of changes in CMRglu, but other tracers can be used to investigate the binding affinity of selected compounds, and how it is modified by the administration of psychedelics. Vollenweider and colleagues studied how psilocybin modulated the in vivo binding of radioactive $\left[{ }^{11} \mathrm{C}\right]$ raclopride to dopamine $\mathrm{D}_{2}$ receptors, finding decreases in binding affinity located in the caudate nucleus and the putamen (Vollenweider et al., 1999). These decreases were attributed to competition with increased levels of endogenous dopamine, suggesting that stimulation of serotonin receptors $5-\mathrm{HT}_{2 \mathrm{~A}}$ and $5-\mathrm{HT}_{1 \mathrm{~A}}$ by psilocybin leads to striatal dopamine release. Finally, PET imaging can be used to map the density of 5$\mathrm{HT}_{2 \mathrm{~A}}$ receptors (e.g. using $\left[{ }^{11} \mathrm{C}\right] \mathrm{Cimbi}-36$ as a radioligand) (Ettrup et al., 2014; Ettrup et al., 2016), the active site of serotonergic psychedelics, yielding a fronto-temporo-parietal distribution that presents some overlap with networks associated with conscious information access and self-referential thought (Rees et al., 2002; Koch et al., 2016; Davey et al., 2016). The reviewed articles using PET and SPECT to investigate changes in brain metabolism under the effects of psychedelics reported results consistent with the distribution of $5-\mathrm{HT}_{2 \mathrm{~A}}$ receptors, agreeing in the observation of increased frontal and parietal metabolism.

\section{Changes in spontaneous activity and functional connectivity}

fMRI provides excellent spatial resolution $(\approx 1 \mathrm{~mm})$ combined with a temporal resolution that is acceptable for many applications $(\approx 1 \mathrm{~s})$. Currently, fMRI is the predominant imaging method for functional brain mapping and for the study of spontaneous brain activity and statistical associations between activity time series measured at different brain regions (i.e. functional connectivity). The sequence of physiological changes leading to the BOLD signal is complex and beyond the scope of the present review; however, it is important to note that fMRI provides indirect measurements of brain activity. Increased flow of oxygenated blood is required to meet the metabolic demands required by ion pumps located in the cell membrane. These pumps must operate to restore the membrane potential to its resting value after depolarization and require the hydrolysis of adenosine triphosphate, a chemical reaction that consumes oxygen. fMRI provides a signal related to the presence of oxygenated hemoglobin in the blood and thus it is sensitive to a combination of blood flow and its level of oxygenation (BOLD signal). This signal is delayed for some seconds respect to neuroelectric activity, and its response to a single electrical event is deformed by the hemodynamic response function (Logothetis and Wandell, 2004).

fMRI can be used to investigate changes in blood flow related to sensory stimulation or task execution, or to quantify changes in spontaneous or resting state activity. This section reviews resting state fMRI studies, while the next addresses how psychedelics affect human cognition in task-based fMRI experiments.

The Imperial College group pioneered the use of resting state fMRI under the effects of psychedelics, beginning with psilocybin (Carhart-Harris et al., 2012). This study produced an interesting but counter- 
intuitive result: psilocybin decreased cerebral blood flow in several regions, including the thalamus, putamen, hypothalamus, posterior cingulate cortex, precuneus, bilateral angular gyrus, supramarginal gyrus. anterior cingulate cortex, frontoinsular cortex and orbitofrontal cortex, among others. These regions present partial overlap with the default mode network (DMN), a fronto-parieto-temporal network associated with spontaneous (i.e. as opposed to elicited) thought and introspection (Raichle, 2015). These changes in cerebral blood flow correlated with the intensity of the reported subjective effects of psilocybin, leading the authors to hypothesize an effect akin to the disengagement of a "reducing valve" associated with the key hubs of the DMN. The concept of a scrambling or dysregulating effect of psychedelics can be traced to this publication, and was subsequently developed by the Imperial College group in following articles.

Further analysis of the psilocybin fMRI data provided evidence supporting the aforementioned hypothesis. Psilocybin increased the variability of BOLD oscillations within the limbic system, leading to an enhanced repertoire of functional connectivity motifs and increased disorder (i.e. entropy) of the time series representing the temporal alternation of these motifs (Tagliazucchi et al., 2014). Petri and colleagues applied more sophisticated methods to show consistent results, with psilocybin reducing the stability of transient functional connectivity patterns, creating an ampler repertoire of less stable functional connectivity motifs (Petri et al., 2014). These articles, together with others to be mentioned below, led Carhart-Harris and colleagues to propose that the subjective effects of psychedelics can be understood in terms of increased entropy of brain activity fluctuations (Carhart-Harris et al., 2014; Carhart-Harris, 2018). Other re-analyses of the 2012 psilocybin fMRI experiment showed increased functional connectivity between DMN and task-positive regions (Carhart-Harris et al., 2013), and generally increased coupling between groups of regions that synchronize during rest, termed resting state networks (RSN) (Roseman et al., 2014).

Experiments with other psychedelic drugs yielded results partially consistent with those of Carhart-Harris et al., 2012. Ayahuasca also decreased the fMRI signal at key DMN hubs and disrupted the functional connectivity of the posterior cingulate cortex (Palhano-Fontes et al., 2015). A multimodal investigation of the effects of LSD by the Imperial College group found increased blood flow and functional connectivity of the visual cortex, correlating with reported changes in visual imagery (Carhart-Harris et al., 2016). Functional connectivity decreased between the parahippocampal gyrus and the retrosplenial cortex, with the prefrontal cortex and the posterior cingulate cortex, and within regions belonging to the DMN. The global functional connectivity of fronto-parietal regions increased under LSD, these increases were also found for psilocybin, presented a significant overlap with maps of 5- $\mathrm{HT}_{2 \mathrm{~A}}$ receptor density, and correlated with the self-reported intensity of ego dissolution (Tagliazucchi et al., 2016) - complementing the functional connectivity correlates under psilocybin previously reported in Lebedev et al., 2015.

It is important to note that a recent article reported findings seemingly in contradiction with those reported in Tagliazucchi et al., 2016; namely, reduced global functional connectivity of fronto-parietal regions under LSD (Preller et al., 2018). However, direct comparison of both results is difficult given the application of global signal regression by Preller and colleagues. Removal of the global signal is known to shift the distribution of BOLD signal correlations towards balanced positive/negative correlations (Murphy et al., 2009); thus, this preprocessing step could have displaced positive correlations of frontoparietal regions towards negative values. Also, in line with Tagliazucchi et al., 2016, Muller and colleagues demonstrated increased functional connectivity between connectivity hubs (i.e. thalamus, striatum, precuneus, anterior cingulate cortex) under the acute effects of LSD (Muller et al., 2018). 
Another study by the same group also showed that functional connectivity increases under LSD correlate with drug-induced hallucinations (Muller et al., 2017).

While data collection is relatively straightforward in resting state fMRI experiments, analyses are frequently highly exploratory, incorporating novel and more sophisticated methods to extract meaningful information from high dimensionality spatiotemporal data. Some examples mentioned in the previous paragraphs include Petri et al., 2014 and Tagliazucchi et al., 2014. Atasoy and colleagues applied connectome-harmonic decomposition to investigate dynamic changes in brain states under LSD, and found an expansion in the repertoire of active brain states (Atasoy et al., 2017). Lord and colleagues investigated how the dynamical exploration of the repertoire of brain networks at rest is modulated by psilocybin (Lord et al., 2019), finding a destabilization of frontoparietal subsystems under psilocybin, consistently with Petri et al., 2014. All studies mentioned in this and previous paragraphs investigated functional connectivity between BOLD signals, and thus were incapable of estimating causal relationships between them. Preller and colleagues applied dynamic causal modeling to resting-state fMRI data acquired during the acute effects of LSD, and found that LSD increased effective connectivity from the thalamus to posterior cingulate cortex in a way directly modulated by serotonin $5-\mathrm{HT}_{2 \mathrm{~A}}$ receptor activation, and decreased effective connectivity from the ventral striatum to the thalamus independently of serotonin $5-\mathrm{HT}_{2 \mathrm{~A}}$ receptor activation. This study adds support to the thalamic filter model, suggesting that the core effects of psychedelics result from gating deficits within cortico-striato-thalamo-cortical feedback loops. Finally, computational modeling has been applied to link functional connectivity changes under LSD with 5-HT ${ }_{2 \mathrm{~A}}$ receptor density (Deco et al., 2018). By combining anatomical data from diffusion magnetic resonance imaging and fMRI with $5-\mathrm{HT}_{2 \mathrm{~A}}$ receptor density maps obtained using PET imaging, the authors constructed a semi-empirical dynamical mean-field quantitative description of populations of excitatory and inhibitory neurons, which allowed them to identify the mechanisms for the non-linear interactions between fMRI data and serotonin receptor activation.

\section{Changes in cognition informed by task-based experiments}

Psychedelics induce idiosyncratic changes in the way the world is perceived and acted upon. The most salient features of the altered state of consciousness relate to perception (Kometer and Vollenweider, 2016); accordingly, the first EEG studies performed under the effects of LSD investigated extensively the modulation of visual and somatosensory evoked potentials. However, the effects of psychedelics extend far beyond the sensory domain and affect other aspects of human cognition. Understanding the cognitive modifications induced by psychedelics, together with their neurophysiological underpinnings, remains one of the most fascinating challenges in the field.

Protocols such as event-related and block designs are, in combination with BOLD fMRI, very powerful tools to map the functional changes associated with task execution and sensory stimulation. In the study of psychedelics, the design of tasks is frequently influenced by the psychotomimetic hypothesis. Deficits in attentional functions observed in schizophrenic patients led Daumann and colleagues to investigate the cerebral correlates of alertness under the effects of DMT and ketamine, a glutamatergic dissociative presenting some overlaps with psychedelics in terms of subjective effects. DMT led to decreased BOLD response during the performance of an alertness task (especially in regions related to visual processing) (Daumann et al., 2010). In a different study, they investigated the effects of DMT in the neural correlates underlying orienting of attention, finding significant behavioral effects without changes in brain activation 
(Daumann et al., 2008). A related experiment investigated the effect of LSD on response inhibition networks using a Go/No-Go task, finding that LSD impaired inhibitory performance and reduced brain activation in the right middle temporal gyrus, superior/middle/inferior frontal gyrus and anterior cingulate cortex and in the left superior frontal and postcentral gyrus and cerebellum (Schmidt et al., 2018).

The Basel and Zurich groups investigated how psychedelics affect emotion, social cognition, and the processing of fearful stimuli. This research is relevant both for the psychotomimetic model (since schizophrenics suffer from social and emotional deficits) and for the potential therapeutic use of psychedelics in certain psychiatric conditions such as depression and anxiety. In this domain, current evidence suggests that psychedelics act by modulating the activation and functional connectivity of the amygdala, a deep brain structure related to the processing of emotional stimuli. LSD reduced reactivity of the left amygdala and the right medial prefrontal cortex during the presentation of fearful faces, with a negative correlation between the activation of the amygdala and the subjective effects of the drug (Mueller et al.,2017). The application of dynamic causal modeling to fMRI data acquired under the effects of psilocybin revealed that the drug decreased the threat-induced modulation of top-down connectivity from the amygdala to primary visual cortex, suggesting a mechanism underlying emotional regulation during the psychedelic state (Kraehenmann et al., 2016). Another study from the same group found that amygdala reactivity to negative and neutral stimuli was lower after psilocybin administration, with changes in reactivity correlated with psilocybin induced-increases in positive mood state (Kraehenmann et al., 2015). Grimm and colleagues investigated the effects of psilocybin on emotion processing networks by assessing the effects on the functional connectivity of the amygdala during a face discrimination task (Grimm et al., 2018). They found that psilocybin decreased the connectivity between the amygdala and the striatum during angry face discrimination, and increased the connectivity between the amygdala and the frontal pole during happy face discrimination. Importantly, Roseman and colleagues investigated the response of the amygdala to neutral, fearful and happy faces in a group of depressive patients who received a single dose of psilocybin with psychological support (Roseman et al., 2018). Increased responses to fearful and happy faces were observed in the right amygdala post-treatment, and these differences predicted clinical improvement at 1-week. Taken together, these studies suggest that psilocybin -as opposed to other drugs used to treat mood disorders- do not blunt the emotional response, but allow patients to successfully confront their emotions.

Further evidence supporting the therapeutic mechanism of action of psilocybin emerges from a study addressing how the drug modulates the processing of negative social interactions, e.g. social rejection. Psilocybin led to reduced feelings of social exclusion, presenting correlations with decreased anterior cingulate cortex and middle frontal gyrus activation, both regions involved in the processing of social pain (Preller et al., 2016). The authors proposed that these results are relevant in the context of the social stigma suffered by psychiatric patients.

Also relevant in the context of the therapeutic effects of psychedelics are studies investigating the processing of autobiographical information. Preller and colleagues found that LSD increased the attribution of personal meaning to previously meaningless music, an effect mediated by cortical midline structures (Preller et al., 2017). Carhart-Harris and colleagues found that psilocybin induced activations in the amygdala, hippocampus, putamen, cingulate cortex, precuneus and temporal and frontal poles, among others, when subjects were cued with personal memories (Carhart-Harris et al., 2012). Furthermore, significant positive correlation was observed between vividness and subjective wellbeing in a two-week follow-up. These studies suggest that psilocybin modulate the processing of autobiographical information 
by increasing the response in a distributed network of brain regions, including the amygdala and midline structures.

fMRI has been used to investigate the neural correlates of altered sensory perception elicited by psychedelic drugs, using both simple and complex visual and auditory stimuli. A pioneering study using ayahuasca showed that during an imagery task the drug induced activations in visual regions comparable to those elicited by natural images with the eyes opened, with additional activations in the cuneus, lingual gyrus, parahippocampal gyrus, retrosplenial and frontopolar cortices (de Araujo et al., 2012). Roseman and colleagues combined a retinotopic localizer with functional connectivity analyses to identify the patches of V1 and V3 representing vertical and horizontal meridians of the visual field, and showed that LSD increased connectivity between patches with incongruent specificity (Roseman et al., 2016). This result supports that LSD leads to a functional organization of the visual cortex less dependent on its retinotopic organization. Taken together, these two studies show that psychedelics induced vivid visual imagery related to the activation of visual regions with eyes closed, and that they re-organize the low-level processing of visual information.

The processing of musical stimuli has received attention from two independent experiments. LSD increased the functional connectivity between the parahippocampus and the visual cortex under LSD, with positive correlation with subjective ratings of eyes-closed visual imagery (including autobiographical imagery) (Kaelen et al., 2016). These results support previous links between the parahippocampal cortex, music evoked emotion and psychedelic-induced mental imagery. In a different study, tonality-tracking analysis of BOLD data revealed that LSD alters neural responses in regions associated with low and highlevel musical and auditory processing, as well as in other areas related to memory, emotion and selfreferential processing (Barrett et al., 2018). Both studies converge in the finding that the interaction between music and the psychedelic states transcends networks involved with auditory processing and involves other cognitive systems as well.

\section{Contemporary electrophysiological studies}

Many results from early studies have been revisited and replicated using modern imaging technologies such as MEG and EEG with source estimation. Also, these methods can yield novel insights not available to early researchers. MEG recordings during the acute effects of psilocybin revealed a broadband decrease in oscillatory power, localized in posterior association cortices for 1 to $50 \mathrm{~Hz}$, and in frontal association cortices for 8 to $100 \mathrm{~Hz}$ (Muthukumaraswamy et al., 2013). Consistent with fMRI studies, large decreases in spectral power appeared within the DMN. Computational modeling showed that desynchronization in the posterior hub of the DMN could be explained by increased excitability of deep-layer pyramidal neurons, rich in 5- $\mathrm{HT}_{2 \mathrm{~A}}$ receptors. An independent article hypothesized that decreases in activity and spectral power due to excitation of these neurons may be related to secondary excitation of inhibitory interneurons (Carhart-Harris et al., 2014). MEG recordings showed consistent results for LSD data: broadband decreases in spectral power were observed, with changes in different bands and anatomical locations correlating with the subjective effects (Carhart-Harris et al., 2016). Decreases in occipital alpha correlated with the intensity of visual imagery, decreases in parietal (midline) and temporal alpha and delta correlated with reports of ego-dissolution. This study also showed that LSD increases the peak 
frequency of the alpha rhythm, consistently with early EEG studies (Gastaut et al., 1953; Bradley et al., 1953; Bradley and Elkes, 1957; Goldstein et al., 1963).

An attractive hypothesis is that diminished alpha power under serotonergic psychedelics reflects loss of cortical inhibition. Alpha oscillations have been repeatedly linked to the inhibition of neural processes irrelevant for the current cognitive demands (Klimesch et al., 2007). Loss of inhibition may imply facilitated access to consciousness, for instance, of spontaneous activity fluctuations in visual areas that are otherwise filtered out from awareness. This hypothesis is consistent with reports of correlations between alpha decreases under LSD and increased visual imagery (Carhart-Harris et al., 2016), and has received direct support by a study with ayahuasca and EEG (Valle et al., 2016). Ayahuasca decreased EEG power in the delta, theta and alpha bands; source imaging showed that occipital alpha decreases correlated with the intensity of visual imagery, and pretreatment with a 5- $\mathrm{HT}_{2 \mathrm{~A}}$ receptor antagonist (ketanserin) inhibited both changes in EEG and the correlation with visual imagery. Further studies with ayahuasca using EEG produced novel insights on psychedelic action on the human brain. Riba and colleagues mapped the time series of EEG spectral power changes, finding that ayahuasca decreased absolute power in all frequency bands (most prominently in the theta band), that these changes began as early as 15-30 min, peaked between 45-120 min and returned to baseline levels at 4-6 h. Schenberg and colleagues investigated the time series of spectral power changes as well as the blood level of DMT, betacarbolines and their metabolites, reporting a biphasic effect (Schenberg et al., 2015). After 50 min from ingestion they reported reduced power in the alpha band (located at the parieto-occipital cortex), together with increased gamma power between 75 and 125 min (located at frontal, parietal and central regions). These effects correlated with circulating levels of the active compounds in ayahasca, i.e. DMT, harmine, harmaline and tetrahydroharmine.

An EEG study with psilocybin reproduced the alpha power decreases, and also found that lagged phase synchronization between a network of brain regions correlated with subjective reports of drug-induced spritual experiences (Kometer et al., 2015). Other studies with psilocybin addressed various aspects of cognition. Bernasconi and colleagues investigated the EEG visual evoked potentials elicited by emotional faces, and found that psilocybin modulated the $168-189 \mathrm{~ms}$ poststimulus potential, with a second period of modulation between 211 - $242 \mathrm{~ms}$ poststimulus. Source imaging localized these changes to limbic areas, including amygdala and the parahippocampal gyrus. Convergent evidence from EEG experiments suggests that psilocybin, compared to ketamine, fails to disrupt the potentials evoked by mismatch negativity (Umbricht et al., 2003; Heekeren et al., 2008). A MEG study using LSD, however, found results conflicting with those of the aforementioned articles: LSD decreased the neural response to novel stimuli and increased that for familiar stimuli, and dynamic causal modeling showed that top-down connectivity was modulated under the effects of LSD (Timmermann et al., 2018).

\section{Future directions of research}

The results reviewed in this review present important consistencies in terms of findings across drugs and imaging modalities. However, variability in the results remains, and this variability may indicate promising future lines of research.

All the compounds mentioned in this review (psilocybin, mescaline, DMT and LSD) share agonism at 5$\mathrm{HT}_{2 \mathrm{~A}}$ receptors as a common mechanism of action. However, these molecules bind and act ather 
serotonin receptor sub-types, as well as at receptors of other neurotransmitters and neuromodulators. Preliminary research shows that action at these other sites can influence the subjective effects of psychedelics (Zamberlan et al., 2018). Differences can be found between substituted tryptamines and phenethylamines: the former present higher binding affinities for $1 \mathrm{~A} / 1 \mathrm{~B}$ receptors, while the latter are more selective for 2A receptors (Nichols, 2016). Many of the currently most widespread psychedelics for recreational use belong to the family of substituted phenethylamines, such as 2,5-Dimethoxy-4methylamphetamine (DOM), 2,5-dimethoxy-4-bromophenethylamine (2C-B) and 25I-NBOMe (Shulgin et al., 2011). However, very few neuroimaging studies have addressed the acute effects of mescaline, the prototypical psychedelic phenethylamine. More generally, placebo-controlled neuroimaging studies can fail to reveal changes specific to certain compounds. Future studies could formally compare the neural correlates elicited by psychedelics with different chemical structures and binding affinity profiles. First steps have been performed using multivariate machine learning classifiers trained and tested using different serotonergic psychedelics and ketamine, revealing that changes in alpha power and connectivity are specific to 5-HT $\mathrm{H}_{2 \mathrm{~A}}$ receptor agonists (Pallavicini et al., 2019). Also, the predictions of the entropic brain hypothesis (Carhart-Harris et al., 2014; Carhart-Harris et al., 2018) are corroborated not only for LSD and psilocybin, but also for ketamine (Schartner et al., 2017).

While experiments using independent imaging modalities can provide important information on psychedelic action on the human brain, future studies must combine imaging modalities to overcome certain limitations. Techniques that measure variables related to cerebral blood flow can be affected by vasoconstriction, an established effect of serotonergic psychedelics (Nichols, 2016). Combined EEGfMRI recordings could facilitate the localization of the prominent decreases in the alpha rhythm caused by these compounds. Simultaneous acquisition of fMRI and PET is a promising tool to investigate changes in brain activity related to in vivo measurements of 5- $\mathrm{HT}_{2 \mathrm{~A}}$ receptor occupancy (Madsen et al., 2019).

For all the variety in imaging modalities, experimental protocols, and investigated compounds, the studies cited in the present review are homogeneous in a dimension highly relevant in the context of psychedelic action: the context surrounding the experience. Since psychedelic experiences are highly sensitive to contextual factors ("setting"), we do not know to which extent knowledge gathered in imaging facilities located in hospitals and research centers generalize to natural or "ecological" settings (Carhart-Harris et al., 2018). This is an important topic, since recreational use (and, potentially, future therapeutic use) takes place in extremely different surroundings, such as private houses, ceremonies and retreats, with external influencing factors such as scents, chants and music. Many psychedelic experiences are, in fact, social experiences undertaken by a group and supervised by a guide. While some studies have attempted recordings in natural settings, these have been limited by underdeveloped mobile EEG technology (Hoffmann et al., 2001; Stuckey et al., 2005). However, this technology has advanced considerably in recent years, and appears ripe for field recordings of psychedelic use.

Finally, neuroimaging studies should be employed to investigate the mechanism underlying the therapeutic effects of psychedelics. The promise of this approach is highlighted by a study investigating the changes in fMRI before and after a single dose of psilocybin in patients suffering from depression (Carhart-Harris et al., 2017). Functional imaging could also be used to predict the likelihood of successful treatment, an application of high relevance considering the financial costs and regulatory hurdles involved in clinical research with psychedelic compounds. 


\section{References}

Atasoy, Selen, et al. "Connectome-harmonic decomposition of human brain activity reveals dynamical repertoire re-organization under LSD." Scientific reports 7.1 (2017): 17661.

Barrett, Frederick S., et al. "Serotonin 2A receptor signaling underlies LSD-induced alteration of the neural response to dynamic changes in music." Cerebral Cortex 28.11 (2017): 3939-3950.

Bernasconi, F., Schmidt, A., Pokorny, T., Kometer, M., Seifritz, E., \& Vollenweider, F. X. (2013). Spatiotemporal brain dynamics of emotional face processing modulations induced by the serotonin $1 \mathrm{~A} / 2 \mathrm{~A}$ receptor agonist psilocybin. Cerebral Cortex, 24(12), 3221-3231.

Bradley, P. B., C. Elkes, and J. Elkes. "On some effects of lysergic acid diethylamide (LSD 25) in normal volunteers." Journal of Physiology 121.2 (1953): 50P-51P.

Bradley, P. B., and Joel Elkes. "The effects of some drugs on the electrical activity of the brain." Brain 80.1 (1957): 77-117.

Carhart-Harris, Robin L., et al. "Functional connectivity measures after psilocybin inform a novel hypothesis of early psychosis." Schizophrenia bulletin 39.6 (2012): 1343-1351.

Carhart-Harris, Robin L., et al. "Implications for psychedelic-assisted psychotherapy: functional magnetic resonance imaging study with psilocybin." The British Journal of Psychiatry 200.3 (2012): 238-244.

Carhart-Harris, Robin Lester, et al. "The entropic brain: a theory of conscious states informed by neuroimaging research with psychedelic drugs." Frontiers in human neuroscience 8 (2014): 20.

Carhart-Harris, R., R. Leech, and E. Tagliazucchi. "How do hallucinogens work on the brain." Journal of Psychophysiology71.1 (2014): 2-8.

Carhart-Harris, Robin L., et al. "Neural correlates of the LSD experience revealed by multimodal neuroimaging." Proceedings of the National Academy of Sciences 113.17 (2016): 4853-4858.

Carhart-Harris, Robin L., et al. "Psilocybin for treatment-resistant depression: fMRI-measured brain mechanisms." Scientific reports 7.1 (2017): 13187.

Carhart-Harris, Robin L. "The entropic brain-revisited." Neuropharmacology 142 (2018): 167-178.

Carhart-Harris, Robin L., et al. "Psychedelics and the essential importance of context." Journal of Psychopharmacology 32.7 (2018): 725-731.

Chapman, L. F., and R. D. Walter. "Actions of lysergic acid diethylamide on averaged human cortical evoked responses to light flash." Recent advances in biological psychiatry 7 (1965): 23-36.

Da Silva, Fernando Lopes. "EEG: origin and measurement." EEg-fMRI. Springer, Berlin, Heidelberg, 2009. 19-38.

Davey, Christopher G., Jesus Pujol, and Ben J. Harrison. "Mapping the self in the brain's default mode network." Neuroimage 132 (2016): 390-397.

Daumann, Joerg, et al. "Neuronal correlates of visual and auditory alertness in the DMT and ketamine model of psychosis." Journal of psychopharmacology 24.10 (2010): 1515-1524. 
Daumann, Jörg, et al. "Pharmacological modulation of the neural basis underlying inhibition of return (IOR) in the human 5-HT 2A agonist and NMDA antagonist model of psychosis." Psychopharmacology 200.4 (2008): 573-583.

de Araujo, Draulio B., et al. "Seeing with the eyes shut: neural basis of enhanced imagery following ayahuasca ingestion." Human brain mapping 33.11 (2012): 2550-2560.

Deco, Gustavo, et al. "Whole-brain multimodal neuroimaging model using serotonin receptor maps explains non-linear functional effects of LSD." Current Biology 28.19 (2018): 3065-3074.

dos Santos, Rafael G., et al. "Classical hallucinogens and neuroimaging: A systematic review of human studies: Hallucinogens and neuroimaging." Neuroscience \& Biobehavioral Reviews 71 (2016): 715-728.

Ettrup, Anders, et al. "Serotonin 2A receptor agonist binding in the human brain with [11C] Cimbi36." Journal of Cerebral Blood Flow \& Metabolism 34.7 (2014): 1188-1196.

Ettrup, Anders, et al. "Serotonin 2A receptor agonist binding in the human brain with [11C] Cimbi-36: test-retest reproducibility and head-to-head comparison with the antagonist [18F] altanserin." Neuroimage 130 (2016): 167-174.

Hermle, Leo, et al. "Mescaline-induced psychopathological, neuropsychological, and neurometabolic effects in normal subjects: experimental psychosis as a tool for psychiatric research." Biological psychiatry 32.11 (1992): 976-991.

Hoffmann, Erik, Jan M. Keppel Hesselink, and Y. W. D. S. Barbosa. "Effects of a psychedelic, tropical tea, ayahuasca, on the electroencephalographic (EEG) activity of the human brain during a shamanistic ritual." MAPS Bulletin 11.1 (2001): 25-30.

Horovitz, Z. P., et al. "Behavioral and electroencephalographic effects of LSD." Journal of pharmaceutical sciences 54.1 (1965): 108-110.

Gastaut, H., et al. "Action de la diéthylamide de l'acide d-lysergique (LSD 25) sur les fonctions psychiques et I'électroencéphalogramme." Stereotactic and Functional Neurosurgery 13.2 (1953): 102120.

Goldstein, Leonide, et al. "Quantitative electroencephalographic analysis of naturally occurring (schizophrenic) and drug-induced psychotic states in human males." Clinical Pharmacology \& Therapeutics 4.1 (1963): 10-21.

Goldstein, Leonide, and Neal W. Stoltzfus. "Psychoactive drug-induced changes of interhemispheric EEG amplitude relationships." Agents and actions 3.2 (1973): 124-132.

Gouzoulis-Mayfrank, Euphrosyne, et al. "Neurometabolic effects of psilocybin, 3, 4methylenedioxyethylamphetamine (MDE) and d-methamphetamine in healthy volunteers: a double-blind, placebo-controlled PET study with [18F] FDG." Neuropsychopharmacology 20.6 (1999): 565-581.

Greiner, Theodore, Neil R. Burch, and Robert Edelberg. "Psychopathology and psychophysiology of minimal LSD-25 dosage: A preliminary dosage-response spectrum." AMA Archives of Neurology \& Psychiatry 79.2 (1958): 208-210. 
Grimm, O., et al. "Psilocybin modulates functional connectivity of the amygdala during emotional face discrimination." European Neuropsychopharmacology 28.6 (2018): 691-700.

Heekeren, Karsten, et al. "Mismatch negativity generation in the human 5HT 2A agonist and NMDA antagonist model of psychosis." Psychopharmacology 199.1 (2008): 77-88.

Kaelen, Mendel, et al. "LSD modulates music-induced imagery via changes in parahippocampal connectivity." European Neuropsychopharmacology 26.7 (2016): 1099-1109.

Klimesch, Wolfgang, Paul Sauseng, and Simon Hanslmayr. "EEG alpha oscillations: the inhibition-timing hypothesis." Brain research reviews 53.1 (2007): 63-88.

Kraehenmann, Rainer, et al. "The mixed serotonin receptor agonist psilocybin reduces threat-induced modulation of amygdala connectivity." NeuroImage: Clinical 11 (2016): 53-60.

Kraehenmann, Rainer, et al. "Psilocybin-induced decrease in amygdala reactivity correlates with enhanced positive mood in healthy volunteers." Biological psychiatry 78.8 (2015): 572-581.

Kometer, Michael, et al. "Psilocybin-induced spiritual experiences and insightfulness are associated with synchronization of neuronal oscillations." Psychopharmacology232.19 (2015): 3663-3676.

Kometer, Michael, and Franz X. Vollenweider. "Serotonergic hallucinogen-induced visual perceptual alterations." Behavioral Neurobiology of Psychedelic Drugs. Springer, Berlin, Heidelberg, 2016. 257-282.

Koch, Christof, et al. "Neural correlates of consciousness: progress and problems." Nature Reviews Neuroscience 17.5 (2016): 307.

Lebedev, Alexander V., et al. "Finding the self by losing the self: Neural correlates of ego-dissolution under psilocybin." Human brain mapping 36.8 (2015): 3137-3153.

Logothetis, Nikos K., and Brian A. Wandell. "Interpreting the BOLD signal." Annu. Rev. Physiol. 66 (2004): 735-769.

Lord, Louis-David, et al. "Dynamical exploration of the repertoire of brain networks at rest is modulated by psilocybin." NeuroImage 199 (2019): 127-142.

Madsen, M., et al. "Psychedelic effects of psilocybin correlate with serotonin 2 A receptor occupancy and plasma psilocin levels." (2019).

Michel, Christoph M., et al. "EEG source imaging." Clinical neurophysiology 115.10 (2004): 2195-2222.

Monroe, Russell R., et al. "Correlation of rhinencephalic electrograms with behavior: a study of humans under the influence of LSD and mescaline." Electroencephalography and clinical neurophysiology 9.4 (1957): 623-642.

Monroe, R. R., and R. G. Heath. "Effects of lysergic acid and various derivatives on depth and cortical electrograms." Journal of neuropsychiatry 3 (1961): 75.

Monroe, Russell R. "Drug effects on subcortical electrograms in humans." Psychotropic Drugs and the Human EEG. Vol. 8. Karger Publishers, 1974. 228-237. 
Müller, F., et al. "Increased thalamic resting-state connectivity as a core driver of LSD-induced hallucinations." Acta Psychiatrica Scandinavica 136.6 (2017): 648-657.

Mueller, F., et al. "Acute effects of LSD on amygdala activity during processing of fearful stimuli in healthy subjects." Translational psychiatry 7.4 (2017): e1084.

Müller, Felix, et al. "Altered network hub connectivity after acute LSD administration." NeuroImage: Clinical 18 (2018): 694-701.

Murphy, Kevin, et al. "The impact of global signal regression on resting state correlations: are anticorrelated networks introduced?." Neuroimage 44.3 (2009): 893-905.

Muthukumaraswamy, Suresh D., et al. "Broadband cortical desynchronization underlies the human psychedelic state." Journal of Neuroscience 33.38 (2013): 15171-15183.

Muzio, J., H. Roffwarg, and E. Kaufman. "Alteration in the young adult human sleep EEG configuration resulting from d-LSD-25." Report to Association for the Psychophysiological Study of Sleep, Palo Alto, Calif (1964).

Muzio, Joseph N., Howard P. Roffwarg, and Edward Kaufman. "Alterations in the nocturnal sleep cycle resulting from LSD." Electroencephalography and clinical neurophysiology 21.4 (1966): 313-324.

Nichols, David E. "Psychedelics." Pharmacological reviews 68.2 (2016): 264-355.

Palhano-Fontes, Fernanda, et al. "The psychedelic state induced by ayahuasca modulates the activity and connectivity of the default mode network." PloS one 10.2 (2015): e0118143.

Pallavicini, Carla, et al. "Spectral signatures of serotonergic psychedelics and glutamatergic dissociatives." NeuroImage(2019).

Petri, Giovanni, et al. "Homological scaffolds of brain functional networks." Journal of The Royal Society Interface 11.101 (2014): 20140873.

Preller, Katrin H., et al. "Effects of serotonin 2A/1A receptor stimulation on social exclusion processing." Proceedings of the National Academy of Sciences 113.18 (2016): 5119-5124.

Preller, Katrin H., et al. "The fabric of meaning and subjective effects in LSD-induced states depend on serotonin 2A receptor activation." Current Biology 27.3 (2017): 451-457.

Preller, Katrin H., et al. "Changes in global and thalamic brain connectivity in LSD-induced altered states of consciousness are attributable to the 5-HT2A receptor." Elife 7 (2018): e35082.

Preller, Katrin H., et al. "Effective connectivity changes in LSD-induced altered states of consciousness in humans." Proceedings of the National Academy of Sciences 116.7 (2019): 2743-2748.

Raichle, Marcus E. "The brain's default mode network." Annual review of neuroscience 38 (2015): 433 447.

Rees, Geraint, Gabriel Kreiman, and Christof Koch. "Neural correlates of consciousness in humans." Nature Reviews Neuroscience 3.4 (2002): 261. 
Rodin, E., and E. Luby. "Effects of LSD-25 on the EEG and photic evoked responses." Archives of general psychiatry 14.4 (1966): 435-441.

Roseman, Leor, et al. "The effects of psilocybin and MDMA on between-network resting state functional connectivity in healthy volunteers." Frontiers in human neuroscience 8 (2014): 204.

Roseman, Leor, et al. "LSD alters eyes-closed functional connectivity within the early visual cortex in a retinotopic fashion." Human brain mapping 37.8 (2016): 3031-3040.

Roseman, Leor, et al. "Increased amygdala responses to emotional faces after psilocybin for treatmentresistant depression." Neuropharmacology 142 (2018): 263-269.

Riba, Jordi, et al. "Topographic pharmaco-EEG mapping of the effects of the South American psychoactive beverage ayahuasca in healthy volunteers." British journal of clinical pharmacology53.6 (2002): 613-628.

Riba, Jordi, et al. "Increased frontal and paralimbic activation following ayahuasca, the pan-Amazonian inebriant." Psychopharmacology 186.1 (2006): 93-98.

Sanches, Rafael Faria, et al. "Antidepressant effects of a single dose of ayahuasca in patients with recurrent depression: a SPECT study." Journal of clinical psychopharmacology 36.1 (2016): 77-81.

Schartner, Michael M., et al. "Increased spontaneous MEG signal diversity for psychoactive doses of ketamine, LSD and psilocybin." Scientific Reports 7 (2017): 46421.

Schenberg, Eduardo Ekman, et al. "Acute biphasic effects of ayahuasca." PLoS One 10.9 (2015): e0137202.

Schmidt, A., et al. "Acute LSD effects on response inhibition neural networks." Psychological medicine 48.9 (2018): 1464-1473.

Scott, Gregory, and Robin L. Carhart-Harris. "Psychedelics as a treatment for disorders of consciousness." Neuroscience of consciousness 2019.1 (2019): niz003.

Shagass, Ch, M. Schwartz, and M. Amadeo. "Some drug effects on evoked cerebral potentials in man." Journal of neuropsychiatry 3 (1962): S-49.

Shagass, Charles, and Marvin Schwartz. "Evoked potential studies in psychiatric patients." Annals of the New York Academy of Sciences (1964).

Shagass, Charles. "Effects of LSD on somatosensory and visual evoked responses and on the EEG in man." Recent advances in biological psychiatry. Springer, Boston, MA, 1967. 209-227.

Shulgin, Alexander Theodore, Tania Manning, and Paul F. Daley. The Shulgin index: Psychedelic phenethylamines and related compounds. Transform Press, 2011.

Stuckey, David E., Robert Lawson, and Luis Eduardo Luna. "EEG gamma coherence and other correlates of subjective reports during ayahuasca experiences." Journal of Psychoactive Drugs 37.2 (2005): 163-178.

Tagliazucchi, Enzo, et al. "Enhanced repertoire of brain dynamical states during the psychedelic experience." Human brain mapping 35.11 (2014): 5442-5456. 
Tagliazucchi, Enzo, et al. "Increased global functional connectivity correlates with LSD-induced ego dissolution." Current Biology 26.8 (2016): 1043-1050.

Timmermann, Christopher, et al. "LSD modulates effective connectivity and neural adaptation mechanisms in an auditory oddball paradigm." Neuropharmacology 142 (2018): 251-262.

Valle, Marta, et al. "Inhibition of alpha oscillations through serotonin-2A receptor activation underlies the visual effects of ayahuasca in humans." European Neuropsychopharmacology26.7 (2016): 1161-1175.

Umbricht, Daniel, et al. "Effects of the 5-HT 2A agonist psilocybin on mismatch negativity generation and AX-continuous performance task: implications for the neuropharmacology of cognitive deficits in schizophrenia." Neuropsychopharmacology28.1 (2003): 170.

Vollenweider, F. X., et al. "Positron emission tomography and fluorodeoxyglucose studies of metabolic hyperfrontality and psychopathology in the psilocybin model of psychosis." Neuropsychopharmacology 16.5 (1997): 357-372.

Vollenweider, Franz X., et al. "5-HT modulation of dopamine release in basal ganglia in psilocybininduced psychosis in man - a PET study with [11 C] raclopride." Neuropsychopharmacology 20.5 (1999): 424.

Yanakieva, Steliana, et al. "The effects of microdose LSD on time perception: a randomised, double-blind, placebo-controlled trial." Psychopharmacology 236.4 (2019): 1159-1170.

Zamberlan, Federico, et al. "The varieties of the psychedelic experience: a preliminary study of the association between the reported subjective effects and the binding affinity profiles of substituted phenethylamines and tryptamines." Frontiers in integrative neuroscience 12 (2018): 54. 